\title{
Clinical outcomes of staff training in positive behaviour support to reduce challenging behaviour in adults with intellectual disability: cluster randomised controlled trial
}

Angela Hassiotis, Michaela Poppe, Andre Strydom, Victoria Vickerstaff, Ian S. Hall, Jason Crabtree, Rumana Z. Omar, Michael King, Rachael Hunter, Asit Biswas, Viv Cooper, William Howie and Michael J. Crawford

\section{Background}

Staff training in positive behaviour support (PBS) is a widespread treatment approach for challenging behaviour in adults with intellectual disability.

\section{Aims}

To evaluate whether such training is clinically effective in reducing challenging behaviour during routine care (trial registration: NCT01680276)

\section{Method}

We carried out a multicentre, cluster randomised controlled trial involving 23 community intellectual disability services in England, randomly allocated to manual-assisted staff training in PBS $(n=11)$ or treatment as usual (TAU, $n=12)$. Data were collected from 246 adult participants.

\section{Results}

No treatment effects were found for the primary outcome (challenging behaviour over 12 months, adjusted mean difference $=-2.14,95 \% \mathrm{Cl}:-8.79,4.51$ ) or secondary outcomes.

\section{Conclusions}

Staff training in PBS, as applied in this study, did not reduce challenging behaviour. Further research should tackle implementation issues and endeavour to identify other interventions that can reduce challenging behaviour.

\section{Declaration of interest}

None.

\section{Copyright and usage}

(c) The Royal College of Psychiatrists 2018
Challenging behaviour is common in adults with intellectual disability, has a reported prevalence of $10-15 \%,{ }^{1,2}$ and often leads to long-term admission to hospital, restrictive care practices and neglect. ${ }^{3-5}$ The need for effective treatment options for challenging behaviour is urgent. Positive behaviour support (PBS) is recommended in routine care for adults with intellectual disability who present with challenging behaviour as it has the greatest evidencebased efficacy. PBS is a multicomponent approach focused on reducing challenging behaviour by using behavioural techniques and consequently improving quality of life in individuals with intellectual disability ${ }^{6}$ and other population groups. ${ }^{7-10}$ PBS aims to help professionals and family or carers better understand an individual's behaviour, and to apply personalised approaches to the management of that behaviour. It can be implemented in a number of ways, including via a single practitioner, ${ }^{11-13}$ via professional teams offering interdisciplinary contributions to the PBS framework, ${ }^{14,15}$ and via a system-wide implementation comprising a tiered model of prevention that covers an entire organisation or geographical area. ${ }^{16}$

The only pilot randomised controlled trial (RCT) of PBS incorporating applied behaviour analysis was delivered by a specialist behaviour team in one area in England and it showed promising results by reducing the lethargy and hyperactivity domain scores of the Aberrant Behaviour Checklist-Community (ABC-C). ${ }^{17,18}$ A naturalistic 2-year follow-up of the same trial participants showed a continued positive effect of the intervention compared with treatment as usual (TAU). ${ }^{19}$ Observational studies also showed that training of paid care staff in PBS reduces challenging behaviour. ${ }^{20}$ Evidence indicates that staff competencies are central in treating challenging behaviour, maintaining improvements ${ }^{21}$ and reducing reliance on containment and in-patient care. ${ }^{22,23}$ To the best of our knowledge, although PBS is considered a cornerstone of good quality care internationally, staff in community intellectual disability services may have insufficient skills to deliver it. There are multiple staff training programmes in PBS that show increases in knowledge and perceived confidence in managing challenging behaviour. $^{22}$ This real-world independent multicentre trial investigated the clinical and cost effectiveness of health staff training in PBS in addition to TAU to reduce challenging behaviour in adults with intellectual disability in England. The present article reports the clinical outcomes of the definitive trial. The economic evaluation of the study is in preparation.

The main objective was to compare the clinical effectiveness of staff training in PBS with TAU alone over 12 months. Secondary objectives were to examine (a) the impact of training in PBS in the subgroup with autism spectrum disorders and (b) the interaction between the intervention, gender, level of intellectual disability, presence of mental disorder and challenging behaviour.

\section{Method}

\section{Study design}

The study protocol has been described elsewhere. ${ }^{21}$ In summary, this was a multicentre single-blind, parallel, two-arm cluster RCT of 23 community intellectual disability services in England with active recruitment. 
The study (trial registration: NCT01680276) received ethical approval by the National Research Ethics Service Committee London-Harrow (reference 12/LO/1378).

\section{Service and participant recruitment}

The community intellectual disability services supporting adults with intellectual disability and challenging behaviour (hereafter referred to as clusters) were recruited through the Clinical Research Networks covering urban and semi-rural/rural areas in England. The number of registered adults with intellectual disability in each cluster ranged from 100 to 1000 and services employed a median of 23 full-time equivalent health and/or social care staff (range 4-70). A maximum of 14 participants with intellectual disability aged 18 years and over with any level of intellectual disability (mild to profound) and challenging behaviour as indicated by a total score of at least 15 on the $\mathrm{ABC}-\mathrm{C}^{18}$ were recruited from each cluster. Excluded were (a) participants with a primary clinical diagnosis of personality disorder or substance misuse as there is no evidence that PBS would be a treatment of choice in these instances, participants with a relapse of a pre-existing mental disorder, or where the clinical team decided that a referral to the study would be inappropriate; and (b) clusters which had embedded PBS therapists or local specialist behaviour teams. Health and social care professionals in each cluster identified potential participants who were screened for eligibility and expressed interest to meet with researchers prior to cluster allocation.

Clinical managers in each cluster were asked, and they agreed, to reduce the routine caseload of the staff who volunteered to train by about $30 \%$ to allow them sufficient time to deliver enhanced treatment to the trial participants. This was based on an assumption of spending a total of approximately $12.5 \mathrm{~h}$ on the intervention per participant, excluding travel and paperwork.

Easy read information sheets and consent forms were prepared with assistance from the study service user reference group. Researchers were trained in obtaining informed consent and in the study processes. Where a participant lacked capacity, another adult was identified or nominated to act as consultee on their behalf.

\section{Randomisation and masking}

The clusters were randomised by an independent web-based randomisation system (Sealed Envelope) and random permuted blocks on a 1:1 allocation. We stratified the randomisation by calculating the staff/patient ratio for each cluster, creating a binary factor which indicated whether a cluster was below or above the median ratio. The trial manager contacted the sites to inform them of the treatment allocation.

Researchers conducting the study assessments were blind to arm allocation status. Researchers were asked to guess allocation for each participant at each follow-up point and to report any incident of unblinding.

\section{Procedures}

\section{PBS training}

Two health staff (henceforth referred to as therapists) from a variety of professions - e.g. psychiatrists, psychologists, nurses, occupational therapists, and speech and language therapists - from each cluster volunteered to receive the training. This included three 2-day face-to-face workshops supported by a manual and delivered by an organisation with a track record in training delivery across many clinical settings and a wide consultancy client base.

The curriculum consisted of the following topics that are essential elements of the application of PBS in routine care: (a) functional behavioural assessment and formulation skills, using the Brief Behavioural Assessment Tool for brief functional analyses

(b) primary prevention of challenging behaviour

(c) secondary prevention and reactive strategies

(d) periodic service review and problem solving

(i) developing individualised periodic service reviews

(ii) troubleshooting.

PBS is a combination of approaches which mainly aim at altering aspects of the environment that may have an impact on behaviour. These include understanding the triggers that lead to a behavioural outburst, improvement of communication between individuals and their carers, promotion of a person-centred community living and use of specific techniques to achieve changes in behaviour by encouraging pro-social responses from the individual. Therapists were shown how to (a) fill in behavioural charts; (b) work on developing interventions for each identified behaviour, (c) plan interventions using non-contingent reinforcement, skills teaching and differential reinforcement; and (d) take into consideration the impact of other potential triggers such as ill health. Each participant's plan should, therefore, include some of these aspects after a comprehensive assessment and observations. Two cohorts of therapists were trained over a 15-week period and therapists were expected to have begun work with participants who had completed a baseline assessment after the first workshop. The therapists received a certificate of completion of training. This is an accepted training format deemed appropriate for the study, although variations in duration and content do exist internationally.

Each therapist was allocated one of the four trainers as a mentor for 1 year and the therapists were responsible for using this facility which was aimed at maintaining motivation and enhancing practice skills. However, to ensure an increase in uptake, monthly teleconferences and site visits by trainers and study personnel were conducted, together with the therapists being supported by an administrator in completing and submitting trial-related paperwork.

Clinical responsibility remained with the clusters.

\section{Treatment as usual}

TAU included any treatment approach that is available to community intellectual disability teams within the National Health Service. Most services in England employ a variety of health and social care professionals, and patients have access to behavioural, psychosocial and pharmacological interventions, e.g. physical health checks, simple behavioural modification, and prescribing and monitoring of psychotropic medication. None of those treatments is strongly evidence based but there is sufficient guidance concerning 'what good care looks like'.

All aspects of TAU were also available to the participants in the intervention arm.

In five cases, it was revealed that trial participants lived in accommodation where the provider had offered PBS awareness seminars or employed consultants to advise its care staff on PBS approaches.

The researchers collected participant demographic information (gender, age, and ethnicity), level of intellectual disability (measured by the Wechsler Abbreviated Scale of Intelligence; WASI ${ }^{24}$ and carer-reported adaptive behaviour (measured by the short version of the Adaptive Behaviour Scale ${ }^{25}$ ) at baseline. Cause of intellectual disability was recorded if known. Participants were also screened for autism using the autism symptom checklist of the Mini Psychopathology Assessment Scale for Adults with Developmental Disability (Mini PASADD). ${ }^{26}$ The postcode of the participant's 
residence was recorded for linkage with the Index of Multiple Deprivation, obtained via the UK Data Service website.

Follow-up assessments were conducted at 6 and 12 months after randomisation with a window of \pm 4 weeks around the due date for each assessment.

\section{Outcomes}

The primary outcome was challenging behaviour measured by the total $\mathrm{ABC}-\mathrm{C}$ score $\left(\mathrm{ABC}-\mathrm{C}_{\mathrm{T}}\right)$ over 12 months. ${ }^{18}$ Secondary outcomes were symptoms of mental disorder (Mini PASADD), ${ }^{26}$ Community Participation (Guernsey Community Participation and Leisure Activities Scale), ${ }^{27}$ Family Carer Burden (Uplift/ Burden Scale $)^{28}$ and Family Carer Psychiatric MorbidityGHQ12. ${ }^{29}$ Paid Carer Burden was measured with the Caregiving Difficulty Scale-Intellectual Disability. ${ }^{30}$ Primary and secondary outcome measures were administered to the family or paid carer at all three assessment points.

Serious adverse events were defined as events that were life threatening, resulted in death, hospital admissions/prolongation of a stay in hospital, and/or persistent or significant disability or incapacity.

\section{Statistical analysis}

The sample size was calculated to detect a difference of 0.45 s.d. in the primary outcome, $\mathrm{ABC}-\mathrm{C}_{\mathrm{T}}$ score, measured over 12 months, with $90 \%$ power and $5 \%$ significance level; ${ }^{21}$ indicating that a minimum of 19 clusters and 246 participants were required.

The analysis plan was developed and discussed with the Trial Management Team and further agreed with the Data Monitoring and Ethics Committee and the Trial Steering Committee, which also oversaw the conduct of the study.

\section{Primary outcome}

For the $\mathrm{ABC}-\mathrm{C}_{\mathrm{T}}$ score, a three-level random effects regression model adjusting for baseline $\mathrm{ABC}-\mathrm{C}_{\mathrm{T}}$ score, time period, staff/ patient ratio and effects of clustering by services and repeated measures within participants was used. This random effects model provides valid inferences under the assumption that data are missing at random. The normality assumptions of the residuals were investigated using residual plots. The primary analysis was performed by two statisticians separately to ensure its accuracy.

Pre-specified patient characteristics that were not balanced across the arms, and that were potentially related to the primary outcome, were adjusted for in a supportive analysis.

\section{Secondary outcomes}

Similar analyses were conducted for the secondary outcomes using linear or logistic models, as appropriate for the type of outcome.

\section{Exploratory multivariate analyses}

These examined the effect of staff training in PBS on standardised $\mathrm{ABC}-\mathrm{C}_{\mathrm{T}}$ domains using a three-level multivariate linear regression model where the standardised domains were considered simultaneously within a multivariate framework, allowing for the estimation of intervention effects for multiple outcomes.

\section{Subgroup analyses}

We explored the treatment effect by gender, age groups (categorised into quartiles), level of intellectual disability, ethnicity, autism spectrum disorder and presence of mental disorder.

\section{Sensitivity analyses}

The model included two random effects at the service level, one for each arm. ${ }^{31}$ The primary analysis model included the predictors of missingness as covariates with a 'baseline observation carried forward' analysis to include participants with missing values of the $\mathrm{ABC}-\mathrm{C}_{\mathrm{T}}$ score.

All statistical tests and confidence intervals are two sided. Statistical analysis was performed using STATA software version 14. All analyses were by intention to treat. Results from all supportive analyses are exploratory and presented as estimates with confidence intervals.

\section{Fidelity assessment}

An independent reviewer assessed all treatment documentation submitted by the therapists, including functional assessment, observational data, PBS plan and goodness-of-fit checklist using the Behaviour Intervention Plan Quality Evaluation Scoring Guide II. The tool is designed to evaluate the quality of behaviour intervention planning. Plans are classified as weak, underdeveloped, good or superior.

\section{Results}

Recruitment took place from 2 June 2013 to 24 November 2014. Originally, 28 clusters agreed to take part but 5 dropped out prior to allocation. From the remaining 23 clusters, 11 were allocated to the intervention and TAU arm and 12 to the TAU-alone arm. In the 11 intervention clusters, 21 therapists were trained. Of the 382 potential participants that were screened, $246(64 \%)$ consented to take part. One participant was erroneously consented as she/he did not meet the ABC-C inclusion threshold, and therefore was excluded from the analysis. The median number of participants recruited per cluster was 13 (interquartile range [IQR] 6-14) (CONSORT flow diagram shown in Fig. 1).

A total of $215(87 \%)$ and 225 (92\%) participants completed the 6- and 12-month follow-up, respectively. There was no difference in attrition between the arms (7\% in the intervention and $9 \%$ in the TAU arms, respectively). Table 1 shows the baseline demographic and clinical characteristics of the participants.

At baseline, the mean $\mathrm{ABC}-\mathrm{C}_{\mathrm{T}}$ score in the intervention arm was 61.8 (s.d. 27.7) compared with 68.5 (s.d. 29.0) in the TAU arm. In the intervention arm, $\mathrm{ABC}-\mathrm{C}_{\mathrm{T}}$ reduced to 55.5 (s.d. 32.5 ) at 6 months and to 54.0 (s.d. 32.1) at 12 months. The respective scores in the TAU arm were 60.6 (s.d. 32.6) at 6 months and 59.2 (s.d. 28.8) at 12 months.

The primary model used $439 \mathrm{ABC}-\mathrm{C}_{\mathrm{T}}$ score measurements from 233 participants over the two follow-up time points. The intervention was not statistically significant compared with TAU in terms of the $A B C-C_{T}$ score (adjusted mean difference $-2.4 ; 95 \%$ CI: $-8.7,4.5 ; P=0.528$ ). Details are shown in Table 2 and supplementary Fig. 1, available at https://doi.org/10.1192/bjp.2017.34.

The intracluster correlation coefficient (ICC) for the ABC- $\mathrm{C}_{\mathrm{T}}$ score at the service level was 0.021 (95\% CI: $0.001,0.286)$ and for the repeated measures within participants it was 0.625 (95\% CI: $0.542,0.702$ ).

None of the subgroup analyses showed a significant effect with treatment; estimates of the intervention effect on subgroups are shown in Fig. 2.

A series of analyses was undertaken adjusting for (a) area deprivation, (b) participant or carer respondent, (c) unbalanced baseline characteristics (ethnicity and participant's cohabitant), (d) percentage of participants within each cluster who had at least one element of the intervention, (e) a model including two random effects and (f) imputing missing values with baseline 


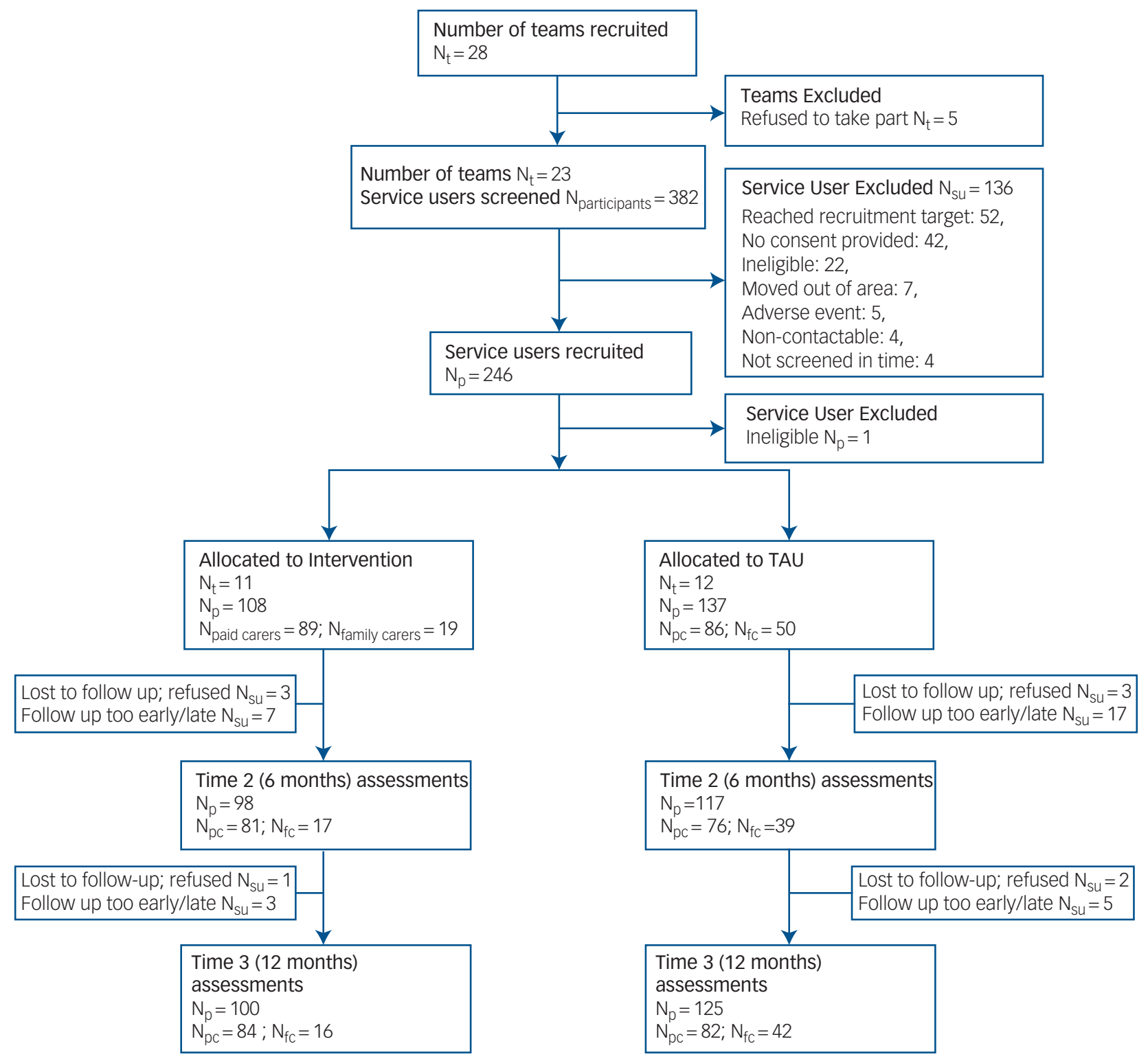

Fig. 1 CONSORT flow diagram.

observation carried forward. All analyses showed non-significant results with differences in $\mathrm{ABC}-\mathrm{C}_{\mathrm{T}}$ score between arms ranging from -3.4 to -0.8 . None of the participant baseline data predicted missing data and, therefore, no further analyses were conducted (supplementary Table 1).

Multivariate analysis examined the effect of the intervention on the individual domains of the ABC-C. The inappropriate speech domain was not included in the multivariate model as it had low correlations ( $\rho=0.300,0.094,0.175,0.360)$ with the (a) irritability, agitation, and crying; (b) lethargy and social withdrawal; (c) stereotypic behaviour; and (d) hyperactivity and non-compliance domains, respectively. The intervention had no significant effect on all four domains (supplementary Table 1).

Regarding the secondary outcomes, there were no differences between the arms for mental ill health or frequency of community activities over 12 months. In total, 69 family carers were included in the study, 19 in the intervention arm and 50 in the TAU arm. The majority $(n=59,86 \%)$ were female with a median age of 54 years (IQR 48-59). Because of the small numbers in the intervention arm, only descriptive analyses were undertaken. A total of
175 paid carers took part in the study, 89 in the intervention arm and 86 in the TAU arm. Two thirds $(n=108,67 \%)$ were female with a median age of 41 years (IQR 32-53). Over the 12 months, $86(49 \%)$ of the paid carers changed (49 in the TAU arm and 37 in the intervention arm, respectively) and therefore no further analyses were carried out (supplementary Table 2).

\section{Psychotropic medication}

A total of $63 \%$ of participants in the intervention arm and $65 \%$ in the control arm were receiving antipsychotic medication by the end of the study. The respective proportions of other psychotropic applications were 72 and $76 \%$, respectively. The proportions of participants on antipsychotics and other psychotropic medications remained stable across the two arms over the study duration.

\section{Serious adverse events}

A total of 29 participants experienced 45 serious adverse events unrelated to the intervention, which were mainly admissions to hospital for a variety of physical ailments and one death. Of these 


\begin{tabular}{|c|c|c|c|}
\hline$N(\%)$ & $\begin{array}{c}\text { Total } \\
(n=245)\end{array}$ & $\begin{array}{c}\text { TAU } \\
(n=137)\end{array}$ & $\begin{array}{c}\text { PBS } \\
(n=108)\end{array}$ \\
\hline \multicolumn{4}{|l|}{ Demographics } \\
\hline Age, years (median, IQR) & $37(25-51)$ & $33(24-51)$ & $42(27-50)$ \\
\hline Gender, male & $157(64)$ & $90(66)$ & $67(62)$ \\
\hline Ethnic origin, White & $176(72)$ & $95(69)$ & $81(75)$ \\
\hline \multicolumn{4}{|c|}{ Service-reported level of intellectual disability } \\
\hline Mild & $41(17)$ & $17(12)$ & $24(22)$ \\
\hline Moderate & $77(31)$ & $46(34)$ & $30(28)$ \\
\hline Severe & $127(52)$ & $73(53)$ & $54(50)$ \\
\hline ABS (median, IQR) & $48(29,68)$ & $42(25,64)$ & $55(35,73)$ \\
\hline $\begin{array}{l}\text { WASI, full-scale } \\
\text { IQ } 4(n=95)\end{array}$ & $44(40,52)$ & $43(40,50)$ & $46(41,53)$ \\
\hline \multicolumn{4}{|l|}{ Current accommodation } \\
\hline Residential & $105(43)$ & $52(38)$ & $53(49)$ \\
\hline Supported living & $69(28)$ & $36(27)$ & $33(30)$ \\
\hline Family home & $64(26)$ & $47(34)$ & $17(16)$ \\
\hline Own flat/house & $7(2)$ & $2(1)$ & $5(5)$ \\
\hline \multicolumn{4}{|l|}{ Clinical } \\
\hline \multicolumn{4}{|l|}{ ABC (median, IQR) } \\
\hline Total score & $64(44,86)$ & $68.5(47,87.5)$ & $60(43,80)$ \\
\hline Irritability & $20(13,29)$ & $21.5(15,29)$ & $18(11,26)$ \\
\hline Lethargy & $12(7,21)$ & $13(6.5,21)$ & $12(7,21)$ \\
\hline Stereotypy & $5(2,10)$ & $5.5(2,10)$ & $4(2,9)$ \\
\hline Hyperactivity & $20(12,26)$ & $21(13,28)$ & $18(11,24)$ \\
\hline Inappropriate speech & $4(1,8)$ & $4(1,8)$ & $5(1,8)$ \\
\hline \multicolumn{4}{|l|}{ Medications } \\
\hline Any medications & $220(90)$ & $124(91)$ & $96(89)$ \\
\hline Antipsychotics & $165(67)$ & $91(66)$ & $74(69)$ \\
\hline Other psychotropic & $180(73)$ & $96(70)$ & $84(78)$ \\
\hline \multicolumn{4}{|l|}{ Mini PASADD } \\
\hline Common mental disorder & $117(49)$ & $61(46)$ & $56(52)$ \\
\hline Severe mental illness & $47(20)$ & $27(20)$ & $20(19)$ \\
\hline Autistic spectrum & $50(21)$ & $31(23)$ & $19(18)$ \\
\hline Physical health problems & $180(74)$ & $107(80)$ & $73(68)$ \\
\hline Mobility $^{\mathrm{a}}(n=180)$ & $64(36)$ & $38(36)$ & $26(36)$ \\
\hline Sensory & $43(24)$ & $29(27)$ & $14(19)$ \\
\hline Epilepsy & 67 (37) & 42 (39) & 25 (34) \\
\hline Incontinence & $78(43)$ & $46(43)$ & $32(44)$ \\
\hline Other & $103(57)$ & $63(59)$ & $40(55)$ \\
\hline
\end{tabular}

serious adverse events, 26 occurred in the intervention arm and 19 in the TAU arm.

Thirteen participants ( 3 in the intervention arm and 10 in the TAU arm) moved from their original address to a new home, either due to closures of previous accommodation or to changes in the participants' needs.

\section{Fidelity of intervention and implementation}

Of the 26 trained therapists, 8 left the study due to long-term illness, maternity leave, sabbatical or job changes. Out of a possible 108 intervention reports, 33 included all elements, i.e. functional assessment, observational data, PBS plan, and goodness-of-fit checklist. Forty-seven included one to three elements, and for 28 participants

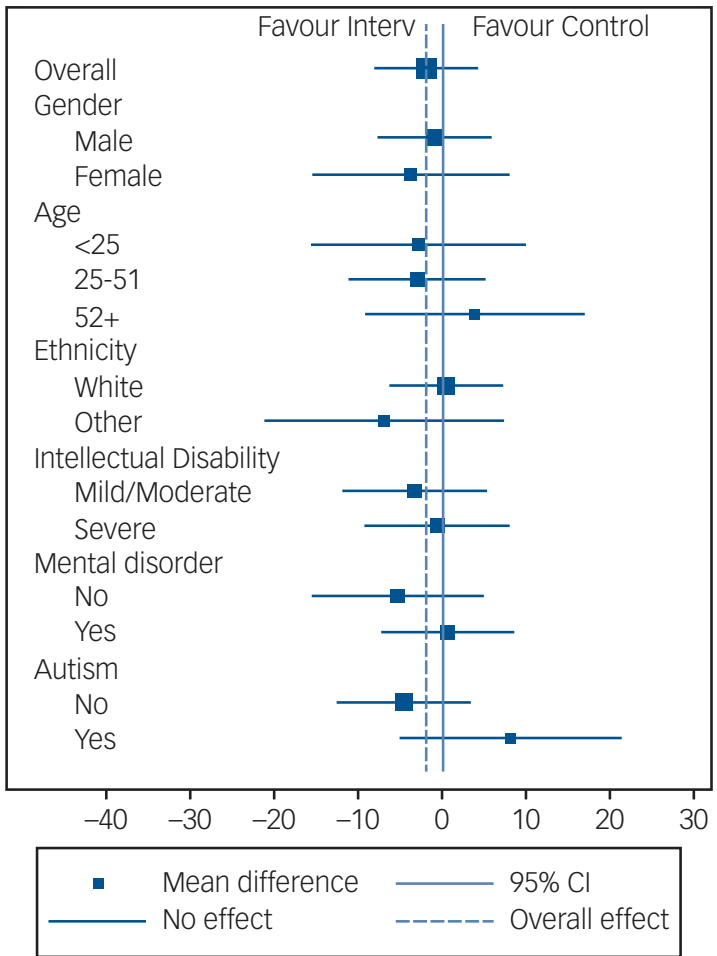

Fig. 2 Subgroup analysis.

there was no submitted paperwork due to the person being not seen, participant's refusal to work with the therapist, not presenting with challenging behaviour at the time of contact, therapist citing lack of time to take on work relating to the study, and a PBS plan having been devised by external providers. The PBS plans included the following domains: 'welcome to my PBS plan', 'understanding my behaviours', 'days that I like', 'primary prevention', 'secondary prevention', 'reactive strategies', and 'evaluation and review' setting the time frame for plan review, usually within 4-6 months.

The available PBS plans were rated as weak by the independent assessor. Weak plans may lead to a change in the identified behaviour, but they lack several of the following: a functional analysis, range of interventions, modelling new approaches and specifying environmental changes that maintain behaviour. Over a 30-month period, the study administrator made weekly to twiceweekly phone calls to the therapists, each intervention site was visited twice, and 22 teleconferences were convened that were attended by $0-4$ therapists and local investigators in addition to trainers and administrators/other study personnel. The therapists rated the training and mentoring arrangements highly, but several reported organisational difficulties, e.g. with obtaining overtime pay for study-related work, dissatisfaction with study-related amount of work in addition to overall caseload, participant not having challenging behaviour or high turnover of paid carers which impeded plan implementation.

\begin{tabular}{|c|c|c|c|c|c|c|}
\hline Time & Arm & $N$ & Mean & s.d. & Median & IQR \\
\hline \multirow[t]{2}{*}{ Baseline } & TAU & 136 & 68.5 & 29.0 & 68.5 & $47-87.5$ \\
\hline & PBS & 107 & 61.8 & 27.7 & 60 & $43-80$ \\
\hline \multirow[t]{2}{*}{6 months } & TAU & 116 & 60.6 & 32.6 & 54 & $37-81$ \\
\hline & PBS & 98 & 55.0 & 32.5 & 50.5 & 30-75 \\
\hline \multirow[t]{2}{*}{12 months } & TAU & 125 & 59.2 & 28.8 & 55 & $42-75$ \\
\hline & PBS & 100 & 54.0 & 32.1 & 49 & $32-73$ \\
\hline
\end{tabular}




\section{Other aspects}

There were six cases of unmasking researchers to the participant's trial arm allocation; another researcher collected data from those sites. Researchers predicted the arm allocation of 123 (59\%) and 126 (56\%) participants at 6 and 12 months, respectively, which were not better than chance.

\section{Discussion}

The cluster RCT evaluated the clinical outcomes of training health professionals - who are specialists in working with adults with intellectual disability - in PBS to reduce challenging behaviour. It did not detect significant reductions in carer-reported challenging behaviour in the intervention plus TAU arm compared with the TAU arm alone over 12 months. Secondary outcomes were also similar between the two arms over 12 months, including the proportion of participants on psychotropic medication. Given the high statistical power, the findings suggest that training the community intellectual disability services staff in PBS, as delivered in this study, was no more effective than TAU in reducing challenging behaviour.

\section{Strengths and limitations}

The study has several strengths, including recruitment of the required number of participants, testing a single primary outcome, achieving a low attrition rate and an a priori analysis plan, which are indicators of a reduced risk of bias. The ICC for the primary outcome is smaller than that which was originally assumed. To guard against the tendency of the impact of training to dissipate over time, we set up long-term mentoring and peer support $^{32}$ as discussed previously. Adjusting for differences in participant characteristics at baseline in the main analysis had no bearing on study outcomes.

The study also has limitations, including the less-than-optimal delivery of the intervention. Of the participants, 30\% (33/108) received all elements of the PBS approach as specified in the training, and $43.5 \%$ received only partial input which was mainly initial observations. Although not all services were able to manage a reduction in the caseloads of therapists, some staff also found the amount of time spent on study-related work to be too onerous. This may be a reflection of the realities of implementing PBS within community intellectual disability services without additional resources, such as specific posts for accredited behavioural therapists.

It could be argued that gradual adoption of PBS-based care in some of the clusters in the TAU arm over the study duration may have reduced any differential between the trial arms. However, we explicitly excluded teams that employed PBS specialists or specialist teams. This was confirmed by a survey of the clusters prior to the study commencing that explored pre-existing behavioural approaches, training, and resources in each cluster. The previous pilot trial ${ }^{17}$ examined a specialist team that included highly motivated and trained behavioural specialists. Therefore, the short duration of training in this study may have been less than optimal in generating confidence in the therapists to deliver a highly complex intervention. Further, because therapists found that some participants did not present with challenging behaviour at the time of contact, the therapists did not initiate any of the intervention procedures. This may be accounted for by the course of challenging behaviour which has a remitting-relapsing nature.

\section{Comparisons with existing literature}

To the best of our knowledge, MacDonald and McGill ${ }^{33}$ conducted the only systematic review to date on outcomes of training staff in
PBS. The authors concluded that the training of paid care staff increases their competence in managing challenging behaviour and reduces the use of restrictive practices and reliance on other professional support, but does not improve participant quality of life. However, none of the included studies used a randomised or quasi-randomised design and follow-up was limited to 6 months. Therefore, previously reported significant effects of staff training in PBS on challenging behaviour are likely due to study bias. ${ }^{34}$ This study did not measure staff skills or knowledge, hence any improvements in those aspects as a consequence of training in PBS were not captured. ${ }^{35,36}$ Therapists may have been less confident in carrying out functional analysis, which is an important element of behavioural approaches; however, multilevel analysis of $n=1$ experimental studies showed that functional analysis does not moderate the relationship between an intervention and its impact on challenging behaviour. Consequently, such an omission is unlikely to have significantly affected participant outcomes. $^{33}$

McClean and $\mathrm{Grey}^{37}$ carried out a 26-month follow-up of a 5 -year rolling training in PBS of paid carers. They found that no specific components of PBS plans were associated with reductions in challenging behaviour. Therefore, even though the plans in this study were rated as weak, they may have had little influence on overall improvements in behaviour. An issue remains, though, as to what are the specific ingredients that would provide added benefit to routine clinical care, given the resource-intensive task of drawing up plans and their subsequent application over time. Other researchers have begun to investigate mindfulness-based PBS training to reduce restrictive practices, improve staff job satisfaction and reduce challenging behaviour in care homes. ${ }^{38}$

As is evident from examining the median scores of the primary outcome, there was a reduction in challenging behaviour for the majority of participants in both arms. Offering training in PBS beyond what is already available within community intellectual disability services does not provide added benefits in reducing challenging behaviour, use of psychotropic medication, or community engagement. Future studies, drawing from psychotherapy research in mental health ${ }^{39}$ should investigate the relative role of setting, participant, therapist and organisational characteristics which underlie any treatment effects found. Finally, identification and evaluation of other treatment approaches are long overdue.

Angela Hassiotis, PhD, University College London Division of Psychiatry, UK and Camden and Islington NHS Foundation Trust, UK; Michaela Poppe, PhD, University College London Division of Psychiatry, UK; Andre Strydom. PhD, King's College London and South London and the Maudsley NHS Foundation Trust, UK; Victoria Vickerstaff, MSc, University College London PRIMENT Clinical Trials Unit, UK; Ian S. Hall, FRCPsych, Jason Crabtree, PhD, Tower Hamlets Community Learning Disability Service, Mile End Hospital, London, UK; Rumana Z. Omar, PhD, Department of Statistical Science, University College London, UK; Michael King, PhD, University College London Division of Psychiatry, UK: Rachael Hunter, MSc, University College London PRIMENT Clinical Trials Unit, UK; Asit Biswas, FRCPsych, Leicestershire Partnership National Health Service Trust, Directorate of Learning Disabilities, Frith Hospital, Leicester, UK; Viv Cooper, OBE, Challenging Behaviour Foundation, UK; William Howie, MRCPsych, Wandsworth Community Mental Health Intellectual Disabilities Team, Springfield Hospital, UK; Michael J. Crawford, PhD, Department of Medicine, Imperial College London, UK.

Correspondence: Angela Hassiotis, University College London Division of Psychiatry, 6th floor, Maple House, 149 Tottenham Court Road, London W1T 7NF, UK. Email: a.hassiotis@ucl.ac.uk

First received 27 Feb 2017, final revision 26 Jun 2017, accepted 14 Nov 2017

\section{Funding}

UK National Institute for Health Research under the Health Technology Assessment Programme (HTA 10/104/13). The study funder had no role in study design, data collection, data analysis, data interpretation, or writing of the report. The corresponding author had full access to all data in the study and had final responsibility for the decision to submit for publication. The views expressed in the article are those of the authors and not necessarily those of 
the National Health Service, National Institute for Health Research or the Department of Health in England.

\section{Acknowledgements}

We thank all study participants and carers who kindly donated their time to complete the study assessments, local and principal investigators and the therapists for their continued support during the study, the members of the study's Data Monitoring and Ethics Committee and the Trial Steering Committee, the Clinical Studies Officers who conducted the study baseline assessments, and the service user study group from the Camden Advocacy Project SURGE for providing valuable advice and feedback throughout the study.

\section{Trial collaborators}

\section{Participating services}

Camden Learning Disability Service; Islington Learning Disability Partnership; Tower Hamlets Community Learning Disability Service; Hackney Learning Disabilities Service; Barnet Learning Disabilities Service; Enfield Integrated Learning Disabilities Service; Barking and Dagenham Learning Disability Service; Havering Learning Disability Service; Waltham Forest Learning Disability Team; Bexley and Greenwich Learning Disability Services; Wandsworth Community Learning Disability Team; Leicester City (East) Community Learning Disability Team; Leicester City (West) Community Learning Disability Team; Charnwood Community Learning Disability Team; Coalville and Hinckley Community Learning Disability Team; Market Harborough, Oadby and Wigston Community Learning Disability Team; Dartford, Gravesend and Swanley Mental Health of Learning Disability Service; Medway Mental Health of Learning Disability Service; East Surrey Community Team for People Learning Disabilities; South West Surrey, North East Hampshire and North West Surrey Community Team for People Learning Disabilities; Mid-Surrey Community Team for People Learning Disabilities; Coventry, South Warwickshire and Rugby Community Learning Disabilities Team; Bradford Learning Disabilities Service.

Recruitment and follow-up: clinical studies officers from the National Institute for Health Resource clinical research networks

Kirsty Collins, Megan Lawrence, Tara Harvey, Naomi Bateman, Karishma Jivraj, Lisa Russell, William Morgan, Sheetal Dandgey, Angeliki Kassari, Hannah Kelly, Michael Kelly, Augustine Musa, Anna Weller, Andra Cosma, Michael Kelly, Neisha Rhule, Sophie Oram, Precina Pankhania, Imogen Sargent, Sarah Dickens, Tessa Saunders, Eliza Jane Corson, Emily Williams, Ruth Charig, Claire Price, Emily Benson, Elizabeth Nyamadzawo and the PBS study research assistants: Natalie Cook, Lisa Dockery, Victoria Ratti and Jessica Blickwedel.

\section{Supplementary material}

Supplementary material is available online at https://doi.org/10.1192/bjp.2017.34.

\section{References}

1 Emerson E, Kiernan C, Alborz A, Reeves D, Mason H, Swarbrick R, et al. The prevalence of challenging behaviours: a total population study. Res Dev Disabil 2001; 22: 77-93.

2 Ali A, Hall I, Blickwedel J, Hassiotis A. Behavioural and cognitive-behavioural interventions for outwardly-directed aggressive behaviour in people with intellectual disabilities. Cochrane Database Syst Rev 2015; 4: CD003406.

3 Webber R, Bowers B, Bigby C. Hospital experiences of older people with intellectual disability: responses of group home staff and family members. J Intellect Dev Disabil 2010; 35: 155-64.
4 The Australian Psychological Society. Evidence-based guidelines to reduce the need for restrictive practices in the disability sector, The Australian Psychological Society, 2011 (https://www.psychology.org.au/Assets/Files/ Restrictive-Practices-Guidelines-for-Psychologists.pdf; accessed December 2016).

5 Allen D, Lowe K, Brophy S, Moore K. Predictors of restrictive reactive strategy use in people with challenging behaviour. J Appl Res Intellect Disabil 2009; 22: 159-68.

6 Gore NJ, McGill P, Toogood S, Allen D, Hughes JC, Baker P, et al. Definition and scope for positive behavioural support. Int J Posit Behav Support 2013; 3: $14-23$.

7 Goh AE, Bambara LM. Individualized positive behaviour support in school settings: a meta-analysis. Remedial Spec Educ 2013; 33: 271-86.

8 Rothwell NA, LaVigna GW, Willis TJ. A non-aversive rehabilitation approach for people with severe behavioural problems resulting from brain injury. Brain Inj 1999; 13: 521-33.

9 Bradshaw CP, Waarsdorp TE, Leaf PJ. Effects of school-wide positive behavioural interventions and supports on child behaviour problems. Pediatrics 2012; 130: e1136-45.

10 Waasdorp TE, Bradshaw CP, Leaf PJ. The impact of schoolwide positive behavioural interventions and supports on bullying and peer rejection: a randomized controlled effectiveness trial. Arch Pediatr Adolesc Med 2012; 166: 149-56.

11 Emerson E, Toogood A, Mansell J, Barrett S, Bell C, Cummings R, et al. Challenging behaviour and community services: I. Introduction and overview. Mental Handicap 1987; 15: 166-9.

12 Toogood S, Bell A, Jaques H, Lewis S, Sinclair C, Wright L. Meeting the challenge in Clwyd: the intensive support team I. Br J Learn Disabil 1994; 22 18-24.

13 Blunden R, Allen D. Facing the Challenge: An Ordinary Life for People with a Learning Disability and Challenging Behaviour. Kings Fund Paper no. 74. Kings Fund Centre, 1987.

14 Allen D, James W, Evans J, Hawkins S, Jenkins R. Positive behavioural support: definition, current status and future directions. Tizard Learn Disabil Rev 2005; 10: 4-11.

15 Sugai G, Horner RH. Defining and describing schoolwide positive behaviour support. In Handbook of Positive Behaviour Support (eds W Sailor, G Dunlap, G Sugai, R Horner). Springer, 2009.

16 Allen D, Kaye N, Horwood S, Gray D, Mines S. The impact of a whole-organisation approach to positive behavioural support on the use of physical interventions. Int J PositBehav Support 2012; 2: 26-30.

17 Hassiotis A, Robotham D, Canagasabey A, Blizard R, Murad S, King M. Randomized, single-blind, controlled trial of a specialist behaviour therapy team for challenging behaviour in adults with intellectual disability. Am J Psychiatr 2009; 166: 1278-85.

18 Aman MG, Singh NN, Stewart AW, Field CJ. The aberrant behaviour checklist: a behaviour rating scale for the assessment of treatment effects. Am J Ment Defic 1985; 89: 485-91.

19 Hassiotis A, Canagasabey A, Robotham D, Marston L, Romeo R, King M. Applied behaviour analysis and standard treatment in intellectual disability: 2-year outcomes. Br J Psychiatry 2011; 198: 490-1.

20 Grey IM, McClean B. Service user outcomes of staff training in positive behaviour support using person-focused training: a control group study. J App/ Res Intellect Disabil 2007; 20: 6-15.

21 Hassiotis A, Strydom A, Crawford M, Hall I, Omar R, Vickerstaff V, et al. Clinical and cost effectiveness of staff training in positive behaviour support (PBS) for treating challenging behaviour in adults with intellectual disability: a cluster randomised controlled trial. BMC Psychiatry 2014; 14: 219.

22 Lowe K, Jones E, Allen D, Davies D, James W, Doyle T, et al. Staff training in positive behaviour support: impact on attitudes and knowledge. J Applied Res Intellect Disabil 2007; 20: 30-40.

23 Beadle-Brown J, Mansell J, Whelton B, Hutchinson A. People with intellectual disability in 'out-of-area' residential placements: 2 . Reasons for and effects of placement. J Intellect Disabil Res 2006; 50: 845-56.

24 Wechsler D. Wechsler Abbreviated Scale of Intelligence - Second Edition (WASIII). Psychological Corporation, 2011.

25 Hatton C, Emerson E, Robertson J, Gregory N, Kessissoglou S, Perry J, et al. The adaptive behaviour scale-residential and community (part I): towards the development of a short form. Res Devl Disabil 2001; 22: 273-88.

26 Prosser H, Moss S, Costello H, Simpson N, Patel P, Rowe S. Reliability and validity of the Mini PAS-ADD for assessing psychiatric disorders in adults with intellectual disability. J Intellect Disabil Res 1998; 42: 264-72.

27 Baker PA. Measurement of community participation and use of leisure by service users with intellectual disability: the Guernsey community 
participation and leisure assessment. J Applied Res Intellect Disabil 2000; 13 169-85.

28 Pruchno R. The effects of help patterns on the mental health of spouse caregivers. Res Ageing 1990; 12: 57-71.

29 Goldberg D, Williams P. A User's Guide to the General Health Questionnaire. NFER-Nelson, 1988

30 McCallion P, McCarron M, Force LT. A measure of subjective burden for dementia care: the caregiving difficulty scale - intellectual disability. $J$ Intellect Disabil Res 2005; 49: 365-71.

31 Omar RZ, Thompson SG. Analysis of a cluster randomized trial with binary outcome data using a multi-level model. Stat Med 2000; 19: 2675-88.

32 Livingston L, Kelly L, Lewis-Holmes E, Baio G, Morris S, Patel N, et al. Nonpharmacological interventions for agitation in dementia: systematic review of randomised controlled trials. Br J Psychiatry 2014; 205: 436-42.

33 MacDonald A, McGill P. Outcomes of staff training in positive behaviour support: a systematic review. J Dev Phys Disabil 2013; 25: 17-33.

34 Heyvaert M, Maes B, Onghena P. A meta-analysis of intervention effects on challenging behaviour among persons with intellectual disability. J Intellect Disabil Res 2010; 54: 634-49.
35 Deveau R, McGill P. Leadership at the front line: impact of practice leadership management style on staff experience in services for people with intellectual disability and challenging behaviour. J Intellect Dev Disabil 2014; 39: 65-72.

36 Hutchinson LM, Hastings RP, Hunt PH, Bowler CL, Banks ME, Totsika V. Who's challenging who? Changing attitudes towards those whose behaviour challenges. J Intellect Disabil Res 2014; 58: 99-109.

37 McClean B, Grey I. A component analysis of positive behaviour support plans. J Intellect Dev Disabil 2012; 37: 221-31.

38 Singh NN, Lancioni GE, Karazsia BT, Myers RE. Caregiver training in mindfulnessbased positive behaviour supports (MBPBS): effects on caregivers and adults with intellectual and developmental disabilities. Front Psychol 2016; 7: 98.

39 Johnsen TJ, Friborg 0 . The effects of cognitive behavioral therapy as an antidepressive treatment is falling: a meta-analysis. Psychol Bull 2015; 141: 747-68. 